\title{
A new subtype-specific monoclonal antibody for IAP-survivin identifies high-risk patients with diffuse large B-cell lymphoma and improves the prognostic value of bcl-2
}

\author{
TRYFONIA MAINOU-FOWLER ${ }^{1}$, LYNN MARIE OVERMAN ${ }^{2}$, HELEN DIGNUM ${ }^{1}$, KATRINA WOOD $^{2}$, \\ STEPHEN CROSIER $^{1}$, BRIAN ANGUS ${ }^{2}$, STEPHEN JOHN PROCTOR $^{1}$ and JOHN J. ANDERSON ${ }^{1}$ \\ Departments of ${ }^{1}$ Haematology and ${ }^{2}$ Pathology, School of Clinical and Laboratory Sciences, \\ William Leech Building, Medical School, Framlington Place, University of Newcastle-upon-Tyne, UK
}

Received July 11, 2007; Accepted September 9, 2007

\begin{abstract}
Anti-apoptotic factors including IAP-survivin and bcl-2 are involved in carcinogenesis and predict for disease outcome for patients with cancer. We used RT-PCR and specific primers to generate two recombinant IAP-survivin proteins; one encoding for the full-length protein and the second comprising the survivin sequence incorporating amino acids 98 to 142 . Both proteins were used to immunize mice and as capture antigens to screen NS1/immune splenocyte hybridoma supernatants for anti-survivin antibody in ELISA assays. The antibody designated F2-9C3 was most effective and reacted with both recombinant proteins and with the native protein present in lysates of A549 (lung carcinoma) and Jurkat cells in Western blots, immunoprecipitation and formalin-fixed tissue sections. Immunohistochemical staining of normal and neoplastic tissues showed association of the F29C3 antibody with the mitotic spindles. Expression of survivin was not detected elsewhere in sections of normal tissue while all neoplastic tissues examined, including those from patients with diffuse large B-cell lymphoma (DLBCL), showed significant expression of survivin. The intensity and localization of staining in these tumours varied and was observed in cytoplasm and/or nuclei. High nuclear expression of survivin predicted the disease outcome in patients with DLBCL. This association was evident when relating intensity to patient survival $(\mathrm{p}=0.0321)$ and strengthened when a score was calculated based on both staining intensity and the proportion of the reactive tumour cells $(\mathrm{p}=0.0128$; reduction in the mean survival times: $35 \%$ and $46 \%$, respectively). Elevated expression of bcl-2 protein also identified the high-
\end{abstract}

Correspondence to: Dr J.J. Anderson or Dr T. Mainou-Fowler, Department of Haematology, School of Clinical and Laboratory Sciences, William Leech Building, Medical School, Framlington Place, University of Newcastle-upon-Tyne, Newcastle-upon-Tyne, NE2 4HH, UK

E-mail: j.j.anderson@ncl.ac.uk tryfonia.mainou-fowler@ncl.ac.uk

Key words: monoclonal antibody, IAP-survivin, bcl-2, diffuse large B-cell lymphoma, prognosis risk patients $(\mathrm{p}=0.0095$; reduction in mean survival time: $37 \%$ ). Over-expression of both factors was a more powerful indicator of poor prognosis than either marker alone $(\mathrm{p}=0.0054,70 \%$ reduction in mean survival time). In conclusion, our novel F2-9C3 monoclonal antibody is effective in determination of expression of IAP-survivin in neoplastic tissue. Nuclear overexpression of IAP-survivin using this antibody predicts the disease outcome in patients with DLBCL and significantly improves the predictive power of bcl-2 in these patients.

\section{Introduction}

Apoptosis refers to the mechanism of programmed cellular demise, a process that can be activated in response to a number of physiologically relevant and diverse stimuli (1). Accumulating evidence suggests that the de-regulation of apoptosis and disturbances of the cell cycle are involved in carcinogenesis. Inhibitors of apoptosis contribute to cancer by aberrantly prolonging cell survival, facilitating the accumulation of transforming mutations and promoting resistance to immunosurveillance (2).

Regulators of apoptosis identified to date include members of the bcl-2 family of proteins known to encompass both proand anti-apoptotic molecules (3). The $b c l-2$ gene has been implicated in the development of cancer and it promotes cellular survival rather than proliferation by inhibiting apoptosis. The $b c l-2$ gene was first discovered by virtue of its involvement in the $\mathrm{t}(14 ; 18)$ chromosomal translocation commonly found in follicular lymphoma (4). De-regulation of the gene induces overexpression of $b c l-2$ mRNA and the encoded protein, a phenomenon which has been observed in many solid cancers (reviewed in ref. 5). Overexpression of bcl-2 protein also occurs in some haematological malignancies (5) and has been associated with poor disease outcome in patients with Hodgkin's lymphoma (6), acute myeloid leukemia (5) and high-grade non-Hodgkin's lymphoma (NHL) (7-9).

Another molecule able to influence the apoptotic balance in cancer has been identified as survivin (10). Survivin is the smallest member of the inhibitors of apoptosis protein (IAP) family (11). It is active when dimerized and has bi-functional activity as it is involved in both cell cycle regulation and 
control of apoptosis (12). Survivin is expressed during mitosis in a cell cycle-dependent manner and it localizes to the spindle micro-tubules in a reaction that requires the inhibition of apoptosis (13). Survivin also inhibits apoptosis directly by binding to and inhibiting the activation of caspases (14) or indirectly by suppressing the activation of specific pro-apoptotic factors involved in the activation of downstream apoptotic pathways (12).

Recent research has shown that the survivin gene encodes multiple alternately spliced mRNAs, which appear to be translated into proteins that exhibit differential sub-cellular localization (15) and apoptotic potential (16). These include the native, full-length anti-apoptotic IAP-survivin, the proapoptotic IAP-survivin 2B, the IAP-survivin DEx3 (16) and the more recently identified variant survivin $3 \mathrm{~B}$ (17).

One of the most significant features of survivin is its differential expression in neoplastic versus normal tissue $(18,19)$. Although expressed during foetal development $(10)$, it is either present in low levels or completely absent in terminally differentiated mammalian cells $(10,11)$. In contrast, it has been found to be overexpressed in a wide variety of tumours $(10,19)$. Elevated levels of IAP survivin in tumour tissue inhibit apop-tosis and thus enhance tumour growth and progression (12) as well as rendering such cells resistant to pro-apoptotic therapies (reviewed in ref. 20). Consequently, overexpression of survivin in tumours has proven useful in clinical diagnosis and in the detection of early relapse in a number of cancers (20). Furthermore, high levels of survivin have been associated with poor prognosis for patients with solid cancers and some haematological malignancies including acute leukaemias and lymphomas (reviewed in ref. 19). Moreover, a recent study has reported that elevated levels of survivin predict for poor disease outcome in patients with diffuse large B-cell lymphoma (DLBCL) (21).

Diffuse large B-cell lymphoma is an aggressive B-cell NHL that accounts for approximately $30 \%$ of all lymphomas in Western countries. With currently used chemotherapy, including CHOP-rituximab, the outcome for patients has greatly improved overall and $40 \%$ of patients enter long-term remission (22). Unfortunately a complete cure cannot be guaranteed with current treatment regimens and approximately $60 \%$ of patients will relapse after a period of remission and die of the disease. There is a clear need to accurately identify the patients who will not experience sustained remission on initial therapy so that an additional or more aggressive alternative therapy might be introduced.

Currently, there is a useful clinical scoring system, the International Prognostic Index (IPI), which although subjective has been developed to identify at diagnosis the patients who may be at high risk of relapse (23). Although the IPI is useful in assessing overall prognosis, it is not sufficiently objective, nor powerful to reliably distinguish the patients who will gain long-term remission with conventional treatment from those who will die from their disease, especially the intermediate risk group.

It is likely that the development of prognostic models which incorporate both clinical and laboratory-based data, such as the expression of biological markers, will allow a more accurate stratification of patients into risk groups. Molecular studies using DNA microarray technology $(24,25)$ and immunohistochemical expression of a number of biological parameters $(8,9,26)$ have stratified patients with DLBCL into different prognostic groups depending on the origin of the malignant cell. However, consensus on the prognostic power of such factors has not yet been reached.

In the present study, we used a novel monoclonal antibody (Mab) to IAP-survivin designated F2-9C3 to examine its expression in tumour tissue from patients with DLBCL and to re-assess its value in assessing prognosis in this group of patients. The study also examines the supplementary prognostic value of determination of bcl-2 protein in the same cohort of patients.

\section{Materials and methods}

Patients. The expression of IAP-survivin and bcl-2 was evaluated retrospectively in a cohort of 155 patients drawn from 436 patients originally enrolled in clinical trials carried out by the Scottish and Newcastle Lymphoma Group and diagnosed with DLBCL between 1984 and 1991. Patient selection was based on the availability of biological material and clinical information. All patients received homogeneous CHOP or CHOP-like therapies (27). Of this population, 53\% were male and $47 \%$ were female with a mean age at diagnosis of 53.46 years (SD, 13.8 years; range, 16-79 years). Of this cohort, 135 patients presented with primary nodal disease and 20 had tumours of primary extranodal origin. The mean survival time from diagnosis, irrespective of the treatment, was 63.01 months. At the end of the trial, $35.5 \%$ were still alive, $63.2 \%$ had died and $2 \%$ were censored as they had died from causes other than DLBCL.

Production of recombinant IAP-survivin proteins. Two recombinant proteins were produced to be used as immunogens and capture antigens to screen hybridoma supernatants in ELISA assays for monoclonal antibody (Mab) production.

Initially, a total RNA extract was prepared from HeLa cells (ATCC: CCL2) using RNAzol B and following standard manufacturer's procedures. RT-PCR was applied to generate a cDNA product incorporating the coding sequence of IAP-survivin. Initially a library was created utilising Superscript II (RNAse $\mathrm{H}^{-}$) (Invitrogen, UK) to perform a reverse transcription reaction primed with random hexamers (pdN6) targeting $5 \mu \mathrm{g}$ of total cellular RNA. The coding sequence of the primary survivin transcript was then amplified by PCR utilising Taq polymerase (Amplitaq Gold, Perkin Elmer UK Ltd) and the following primers; Consensus IAP Sn 5'-GGGATCCCATGGGTGCCCCGACGTTG-3' (a), and Concensus IAP Asn 5'-GGGATCCCTCAATCCAT GGCAGCCAGCTG (b). All survivin RT-PCR reactions were paralleled by amplification of a 350-bp product corresponding to a fragment of the GAPDH housekeeping gene utilising the following primers; GAPDH Sn 5'AGAACATCATCCCTGCCTCC-3' (a), and GAPDH Asn 5'GCCAAATTCGTTGTCATACC-3' (b).

Survivin cDNA was gel purified (Qiagen, UK) and 'AT cloned' into the Eco321 site within the multiple cloning site of the pUC57T plasmid (MBI, Fermentas/Helena BioSciences, UK). Ligation was performed by T4 DNA ligase in the presence of bovine serum albumin and PEG1500. The resultant 
product was transformed into E. coli (Novablue). Transformants were grown on selective media: TAXI-agar plates containing tetracycline $(7 \mu \mathrm{M})$, ampicilin $(150 \mu \mathrm{M}), \mathrm{X}$-gal $(50 \mathrm{mM})$ and isopropyl thiogalactoside (IPTG) $(400 \mu \mathrm{M})$. The constructs containing an appropriately-sized product were identified by colony PCR. Chosen plasmids were then purified and the inserts were sequenced using M13 forward and reverse primers $(\mathrm{MBI})$ in fluorescent dideoxygenic sequencing. Once the identity of the full-length sequence of the cloned insert was established it was excised from pUC 57 via BamH1 digestion, gel-purified and re-ligated into BamH1-cleaved and calf intestinal phosphatase (CIP)-treated $\mathrm{pET}-15 \mathrm{~b}$ vector (Novagen Laboratories, UK). Expansion of the ligated constructs was again performed in T7 polymerase E. coli (Novablue), the successful transformants were selected and expanded in both cultures as explained above. Plasmids were recovered using conventional mini-prep protocols (Qiagen, UK) and the presence, identity and orientation of the respective inserts was confirmed by restriction mapping.

Subsequently, transformation of the construct into T7 polymerase $^{+}$E. coli BL21 (DE3) pLysS allowed IPTG induction and expression of a poly-histidine (his)-tagged survivin molecule. The expressed protein was initially insoluble but was taken into solution in $8 \mathrm{M}$ urea/2 mercaptoethanol (2ME). The protein was re-folded to a soluble form by stepwise dialysis against decreasing concentrations of urea/2ME in TBE buffer. Finally the poly-his-tagged protein was purified by nickel $\left(\mathrm{Ni}^{2+}\right)$ chelation chromatography using his-bind resin in accordance with manufacturer's protocols (Novagen, UK). The protein was eluted from the $\mathrm{Ni}^{2+}$ column in $20 \mathrm{mM}$ Tris- $\mathrm{HCl}, 0.5 \mathrm{M} \mathrm{NaCl}$ and $1 \mathrm{M}$ imidazole, pH 7.9. Finally, imidazole was removed from the recombinant protein by dialysis against imidazole-free elution buffer (500 mM NaCl, 20 mM Tris-HCl, pH 7.9)

A second recombinant protein was made which would encode a unique 45 amino-acid carboxy terminal fragment of the survivin molecule encompassing amino-acids 98 to 142 . Unstable as a poly-his-tagged polypeptide, this fragment was expressed as a glutathione-S-transferase (GST)-fusion protein. The DNA sequence corresponding to this fragment was amplified using the original pUC57-survivin construct as a template, substituting a second sense primer: 5'-GGGATCCC TTGGTGAATTTTTG-3' in combination with the original antisense primer (b) (see above). Once again this allowed cloning of the purified product into pUC57T, the sequence again being confirmed and the insert being excised by restriction digestion and in this instance inserted into the precleaved BamH1 site of pGEX-2T (Pharmacia Biotech, UK). The construct was transformed into E.coli BL21 and chimeric fusion protein expression was induced with IPTG under the control of the tac promoter. Following harvesting, protein was recovered from cell lysates by glutathione-sepharose affinity column chromatography and eluted in $10 \mathrm{mM}$ glutathione. This product was again dialysed against Tris- $\mathrm{HCl}$ to produce a purer protein suitable for use as an immunogen.

Hybridoma generation. Hybridomas were generated following conventional technologies (28) using poly-his-tagged fulllength immunogen- or the GST-tagged fragment-primed Balb/c mouse splenocytes in conjunction with the P3-NS-1/1-Ag-1
(NS-1) mouse myeloma fusion partner (28). Hybridoma colonies picked from cultures fulfilling the requirements of the defined screening procedures were expanded and subjected to additional three successive rounds of limiting dilution cloning ensuring the monoclonality of the final cultures.

Screening and characterisation of monoclonal antibodies reactive with IAP-survivin. The presence of Mab in hybridoma culture supernatants was examined after successive rounds of limiting dilution by ELISA applying an indirect screening method as described previously (28) and using full-length his-tagged recombinant survivin protein and then the GSTtagged survivin fragment as primary and secondary capture antigens. The immobilised anti-survivin antibody was detected via an alkaline phosphatase conjugated secondary antibody detection system using para-nitrophenolphosphate (pNPP) as the chromogenic substrate. The extent of substrate cleavage was determined spectrophotometrically using a Dynex MRX multi-spectrophotometer set to monitor absorbance at $405 \mathrm{~nm}$. Antibody titer was determined as the highest dilution of antigen at which a positive reaction was observed having an optical density (OD) $\geq 3$-fold than the corresponding OD of negative controls lacking the primary antibody.

The specificity of IAP-survivin antibodies was assessed by a combination of discontinuous SDS-PAGE, Western blotting and immunoprecipitation as described previously (28). In Western blots, the survivin antibodies were detected using primary rabbit anti-mouse (Dako, UK) and secondary swine anti-rabbit alkaline phosphatase-conjugated antibodies. Silver staining was employed to visualise immunoprecipitated products (Sigma, UK).

Hybridoma supernatants containing antibody that showed strong reactivity with the full-length and GST capture antigens, as defined by ELISA, were further tested by immunohistochemistry on sections of various normal human tissues including lymph node and a series of human tumours including lymph node biopsies from patients with DLBCL.

Immunohistochemistry (IHC) and scoring. Sections were dewaxed, hydrated prior to blocking of endogenous peroxidase and then stained with the monoclonal anti-bcl-2 (Novocastra) or with the anti-IAP-survivin antibody. Optimal concentrations of each reagent were defined in preliminary experiments which also selected individual optimum antigen-retrieval methods comprising high temperature and pressure (pressure cooking for $1 \mathrm{~min}$ ) in citrate buffer ( $\mathrm{pH} \mathrm{6.0)}$. With respect to survivin, a number of hybrids were selected and screened by IHC and a final clone (designated F2-9C3) was selected based upon its specificity and IHC-reactivity profile. Detection of primary antibody binding was facilitated by the use of the streptavidinbiotin (Dako) detection system while 3,3'-diaminobenzidine (Sigma) served as the chromogen. Finally, the sections were counterstained (Harris' haematoxylin), 'blued', dehydrated and mounted in DPX. Sections without the primary antibody were used as negative controls and normal tonsil as a positive control for both bcl-2 and IAP-survivin, with the latter showing strong specific staining in mitotic figures. Small cell lung carcinoma was also used as a positive control for IAP-survivin.

Tissue sections were scored for both the intensity and the percentage of cells staining. In addition, the localization of 
(a) Recombinant Proteins*

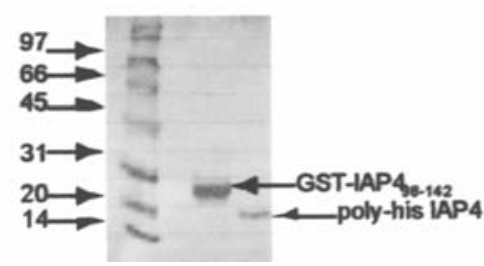

(b) Jurkat'T-cell Lymphoma cells*

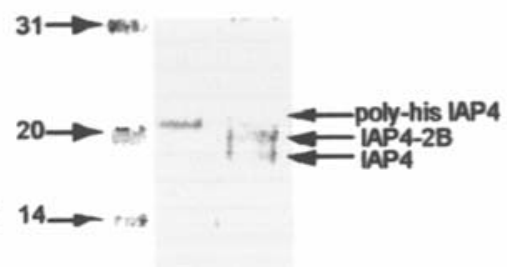

(c) A549 Nocodizole treated Lung Carcinoma Cells*

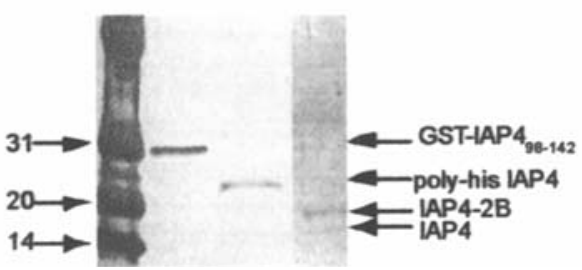

Figure 1. Western blotting and immunoprecipitation with Mab F2-9C3. (a) Panceau red-stained blot of GST-linked IAP-survivin ${ }_{98-142}$ and poly-his-tagged full-length recombinant protein. Track 1, molecular weight marker; track 2, blank; track 3, GST-IAP-survivin ${ }_{98-142}$; and track 4, poly-his-tagged IAP-survivin. (b) Combined Western blotting of immunoprecipitates prepared from RIPA-based lysates of Jurkat T cell lymphoma cells. Track 1, molecular weight marker; track 2, poly-his-tagged full-length recombinant IAP-survivin; and track 3, Jurkat T cell lymphoma cells. (c) Combined Western blotting of immunoprecipitates of: track 1, molecular weight marker; track 2, GST-linked IAP-survivin ${ }_{98-142}$; track 3, poly-his-tagged full-length recombinant IAPsurvivin; and track 4, A549 cells pre-treated with Mab F2-9C3 (cells were pre-treated in cultures with $100 \mathrm{ng} / \mathrm{ml}$ nocodazole and blocked at the G2/M interface) (26). "IAP4 refers to IAP-survivin.

staining was recorded (cytoplasmic/nuclear). No staining was graded as negative, while positive staining was graded on a three point scale (1-3) with positivity of $1^{+}$being set as staining in $20 \%$ of cells (based on a count of 250 cells in multiple fields). For bcl-2, the cut-off point of $\geq 1$ was set as positive. IAP-survivin staining was based on a system analogous to that of the Detre scoring system (29) in which an arithmetic product is calculated as the percentage of cells staining multiplied by the average staining intensity. This gave a range of values between 1 and 300 with a median value of 150. Two groups were identified and defined as low $(<150)$ and high (>150) IAP-survivin-expressing tumours.

Histopathological review. All cases were reviewed by a histopathologist (KW) using haematoxylin and eosin-stained sections and appropriate immunohistochemistry. Only cases fulfilling the WHO criteria for DLBCL (30) were enrolled on the study.

Statistical analysis. All analyses were performed using SPSS 11 version 11.0.4 for MacIntosh (SPSS, Inc., Chicago, IL). Survival analyses were applied in the form of both KaplanMeir analyses and univariate Cox regression. The log-rank test was used to analyse the statistical difference between the groups and a value of $\mathrm{p}<0.05$ was considered to be statistically significant.

\section{Results}

Antibody production. Poly-his-tagged, full-length IAP-survivin and GST-IAP-survivin ${ }_{98-142}$ recombinant proteins were produced from total cellular RNA preparations as described in the Materials and methods. Each served as an effective immunogen when emulsified in Freund's incomplete adjuvant and used to prime Balb/c mice eliciting significant serum antibody responses. Serum titres of immunogen-specific antibody $\geq 1 / 100,000$ were a prerequisite for fusion. Fusions were performed with primed splenocytes from animals inoculated with each different immunogen. Screening of hybridoma culture supernatants by ELISA using full-length IAP-survivin, GST-IAP-survivin ${ }_{98-142}$ or GST-capture antigens indicated that immunization with GST-IAP-survivin ${ }_{98-142}$ produced good serum antibody responses but the animals yielded a few appropriate hybridomas secreting antibody which was reactive with both the C-terminal fragment and the full-length protein. By contrast, splenocytes primed with the poly-his-tagged full-length protein yielded $>10$-fold more hybrids which were reactive with both the homologous fulllength protein and the survivin ${ }_{98-142}$ fragment. Hybridoma lines secreting antibody reactive with both products were further analysed by IHC and antibody staining of paraffin-embedded tissue sections was carefully reviewed. Hybridomas producing antibody showing strong nuclear reactivity and specific staining of the mitotic spindles were expanded and their specificity was analysed by Western blotting and immunoprecipitation. This allowed confirmation of the specific reactivity of the antibodies with both recombinant proteins and native IAP isoforms present in cell lysates. From all the hybridomas examined, a number were subjected to limiting dilution, with re-screening by ELISA and IHC being performed at each round of cloning. Finally the hybridoma clone F2-9C3 was selected, expanded and the IgG2a antibody produced was harvested and used throughout further studies.

Characterization of IAP-survivin by Western blotting and immunoprecipitation. Western blotting and immunoprecipitation experiments confirmed that the survivin antibody designated F2-9C3 reacted with the full-length recombinant poly-his-tagged IAP-survivin protein, of molecular weight of $21 \mathrm{kDa}$, a GST-linked fragment incorporating survivin amino acids 98 to 142 (30 kDa), the native full-length IAP-survivin $(16.4 \mathrm{kDa})$ as well as with the $18.64-\mathrm{kDa}$ IAP-survivin isoform 2B present in cell lysates of A549 cells (small cell lung carcinoma) and Jurkat T-cell lymphoma cells (Fig. 1).

Characterization of IAP-survivin by IHC. Analysis by IHC was carried out on a range of normal and neoplastic tissues as described previously and summarized in Tables I and II. We observed a strong staining of the mitotic figures in both normal (tonsil, Figs. 2A and B, Table II) and neoplastic tissue (Fig. 2C-F). Although the majority of normal adult tissue failed to express survivin (Table I), cerebral cortex, ovary and testis frequently showed weak expression of survivin (Table I). All the tumour tissues examined expressed survivin to various degrees (Table II, Fig. 2C-F). The intensity and localization of staining varied. Over-expression of survivin 
Table I. Immunostaining for IAP-survivin with the F2-9C3 Mab on a range of normal tissues.

\begin{tabular}{|c|c|}
\hline $\begin{array}{l}\text { Normal tissue stained } \\
\text { with F2-9C3 Mab }\end{array}$ & Scoring ${ }^{\mathrm{a}}$ \\
\hline Vein & Negative \\
\hline Stomach & Negative \\
\hline Small intestine & Negative \\
\hline Large intestine & Negative \\
\hline Liver & Negative \\
\hline Kidney & Negative \\
\hline Ureter & Negative \\
\hline Thyroid & Negative \\
\hline Pituitary & Negative \\
\hline Adrenal & Negative \\
\hline Lymph node & Negative \\
\hline Cerebral cortex & $1^{+}$ \\
\hline Skin & Negative \\
\hline Testis & $1^{+}$ \\
\hline Ovary (post men. ${ }^{b}$ ) & $1^{+}$ \\
\hline Ovary (pre men.) & $1^{+}$ \\
\hline Endometrium & Negative \\
\hline Cervix & Negative \\
\hline Cerebellum & Negative \\
\hline Mid-brain & Negative \\
\hline Breast & Negative \\
\hline Lung & Negative \\
\hline Gall bladder & Negative \\
\hline Oesophagus & Negative \\
\hline Tonsil & $\begin{array}{c}2^{+} \\
\text {(mitotic figures in germinal centre cells) }\end{array}$ \\
\hline Prostate & Negative \\
\hline Spinal cord & Negative \\
\hline Skeletal muscle & Negative \\
\hline Bone marrow & Negative \\
\hline
\end{tabular}

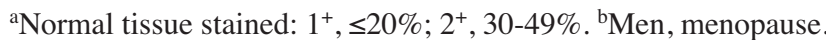

was marked by an intense, generalized nuclear deposition of the chromogen as seen in neuroblastoma sections (Fig. 2D) as well as the strongly positive $\left(3^{+}\right)$DLBCL (Fig. 2F). Low expression of survivin presented as a slight blush of both nuclear and cytoplasmic staining (Fig. 2E). Mitoses were clearly accentuated in all tumours and, particularly, in those with enhanced survivin expression (Fig. 2F).

The anti-apoptotic factors IAP-survivin and bcl-2 are significant prognostic markers in patients with $D L B C L$. In a series of 139 patients with DLBCL, nuclear overexpression of survivin was shown to be an indicator of poor prognosis. Kaplan-Meir analysis of the expression of IAP-survivin based on staining score (0-3) and on the system analogous to that of the Detre scoring system (proportion and intensity, see Materials and methods) revealed that the segregation of the
Table II. Immunostaining for IAP-survivin with the F2-9C3 Mab on a range of neoplastic tissues.

\begin{tabular}{lc} 
Tumour tissues stained with F2-9C3 Mab & Scoring \\
\hline Neuroblastoma & $3^{+}$ \\
Breast fibroadenoma & $2^{+}$ \\
Breast adenoma & $2^{+}$ \\
Breast papilloma & $3^{+}$ \\
Breast DCIS large cell & $3^{+}$ \\
Breast ADH & $4^{+}$ \\
Colon adenoma & $4^{+}$ \\
Colon adenocarcinoma & $2^{+}$ \\
Squamous cell carcinoma of skin & $2^{+}$ \\
Pancreas islet cell adenoma & $2^{+}$ \\
Stomach adenocarcinoma & $3^{+}$ \\
Renal cell carcinoma & $2^{+}$ \\
Oesophagus squamous cell carcinoma & $2^{+}$ \\
Lung adenocarcinoma & $2^{+}$ \\
Squamous cell lung carcinoma & $3^{+}$ \\
Small cell lung carcinoma & $4^{+}$ \\
\hline
\end{tabular}

aTumour tissue stained: $1^{+}, \leq 20 \% ; 2^{+}, 30-49 \% ; 3^{+}, 50-69 \% ; 4^{+}$, $\geq 70 \%$.

two prognostic groups was better employing the Detre scoring system rather than using stain intensity alone $(\mathrm{p}=0.0128 \mathrm{vs}$ $\mathrm{p}=0.0321)$ (Fig. 3A and B). Although there was no difference in the observed mean survival times between the groups of patients with survivin staining score $0 / 1$ and $<150$ (98 vs 90 months, respectively), there was a difference in the survival of patients with scores of $2 / 3$ and $>150$ (64 vs 49 months, respectively). In either case, patients with a low level of survivin expression survived nearly twice as long as those who exhibited high expression.

Similarly, overexpression of bcl-2 predicted for poor disease outcome in the cohort of 152 patients examined (Fig. 3C). Patients whose tumours displayed less than or equal to the median expression (median staining $=1$; see Materials and methods) displayed a mean survival time of 103 months, while in patients with bcl-2 expression greater than the median level of IHC staining the mean survival time was reduced to 65 months. Again, the difference in survival time between the two groups was significant: $\mathrm{p}=0.0095$; logrank value, 6.73 (Fig. 3C).

Further analysis of expression data indicated that overexpression of both IAP-survivin and bcl-2 within the same tumour proved to be an even more powerful indicator of poor prognosis (Fig. 3D). The mean survival time for patients whose tumours expressed both factors was reduced from 120 to only 36 months (70\% reduction). The relative risk was 12.68 with a p-value over the strata of 0.0054 . High expression of survivin in the absence of bcl-2 expression was associated with a reduction of the mean survival time of the patients by $49.5 \%$ while bcl-2 expression in the presence of low levels of survivin reduced the mean survival time by $40 \%$. 

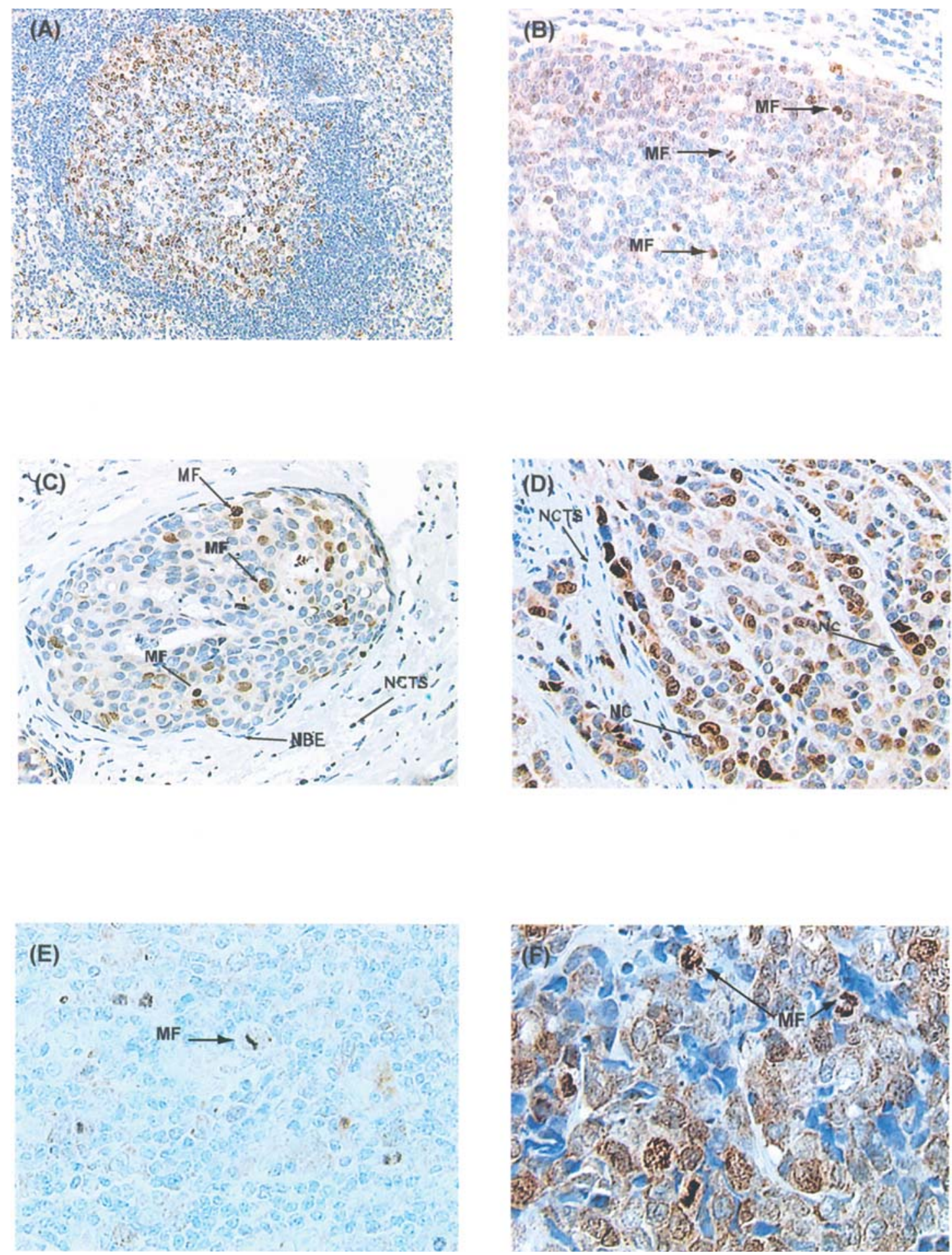

Figure 2. Immunohistochemical staining achieved with the F2-9C3 antibody. Staining of tonsil showing nuclear labelling of an actively dividing germinal centre (A) and clear staining of the mitotic figures (B; arrow heads). Staining of primary breast carcinoma (C) and neuroblastoma (D) tissue sections. The F29C3 Mab produced a strong labelling of the mitotic figures (MF). There was an apparent lack of survivin expression in normal breast epithelium (NBE) and normal connective tissue stroma (NCTS) cells. Strong nuclear overexpression of survivin was observed throughout the nuclei of neuroblastoma cells (NC). Survivin expression was undetectable in normal stroma cells adjacent to the tumour. Staining of high-grade NHL tissue sections with F2-9C3 antibody (E and F). (E) Tumour expressing little survivin but a strong expression of mitotic figures. Few other cells show strong nuclear expression although a slight cytoplasmic blush is evident. A strong nuclear overexpression of survivin with further accentuation of mitosis was observed with another high-grade NHL (F).

\section{Discussion}

In this investigation, we used a novel Mab reactive with the full-length native anti-apoptotic IAP-survivin and the proapoptotic IAP-survivin $2 \mathrm{~B}$ isoform to show that increased nuclear expression of survivin predicts a poor disease outcome in patients with DLBCL. In addition, this is the first study to report that expression of both bcl-2 and IAP-survivin is a more powerful prognostic indicator in DLBCL than either marker alone. 
(A)

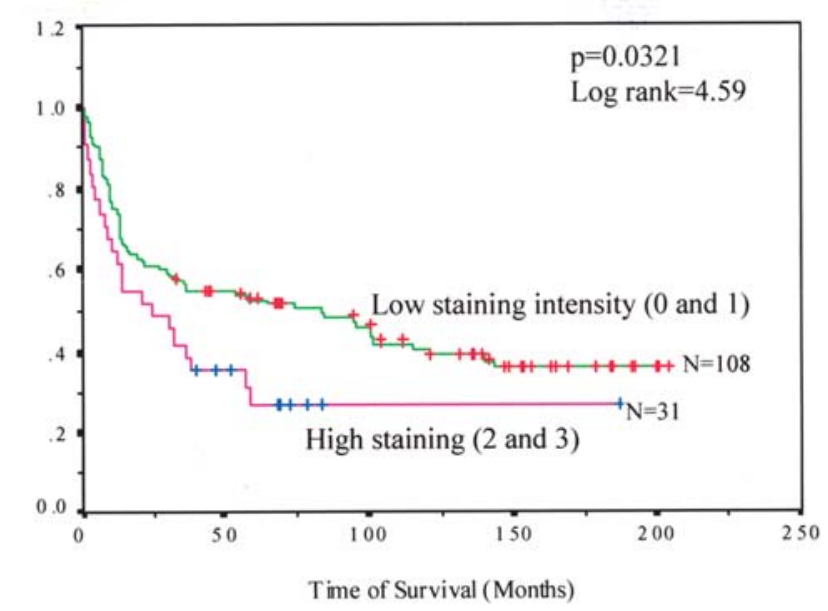

(B)

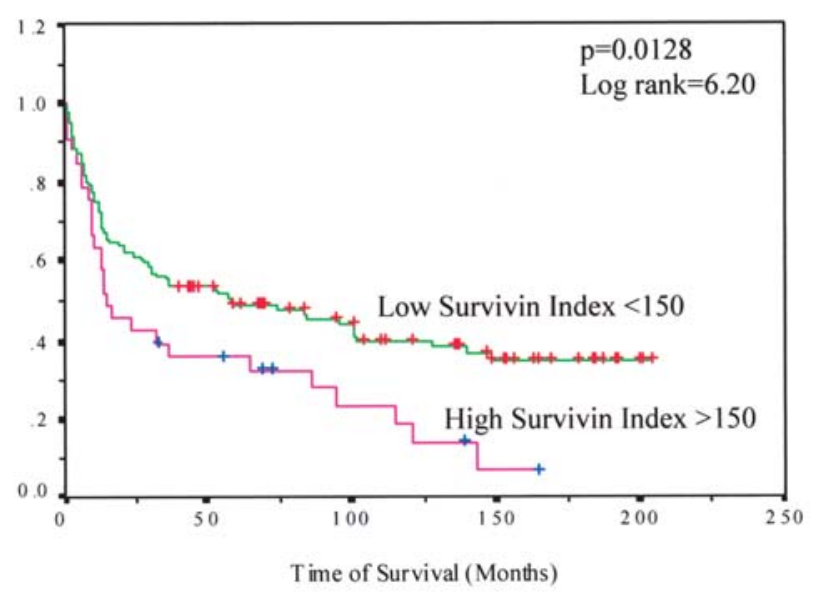

(C)

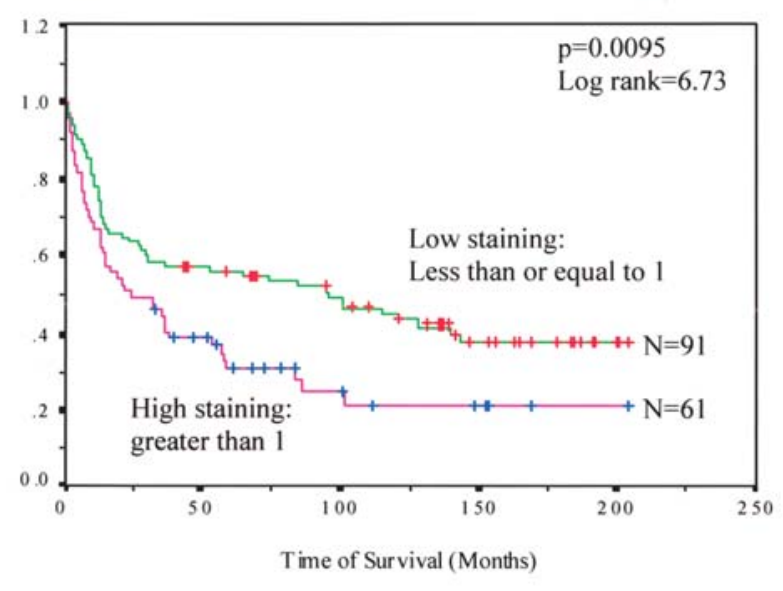

(D)

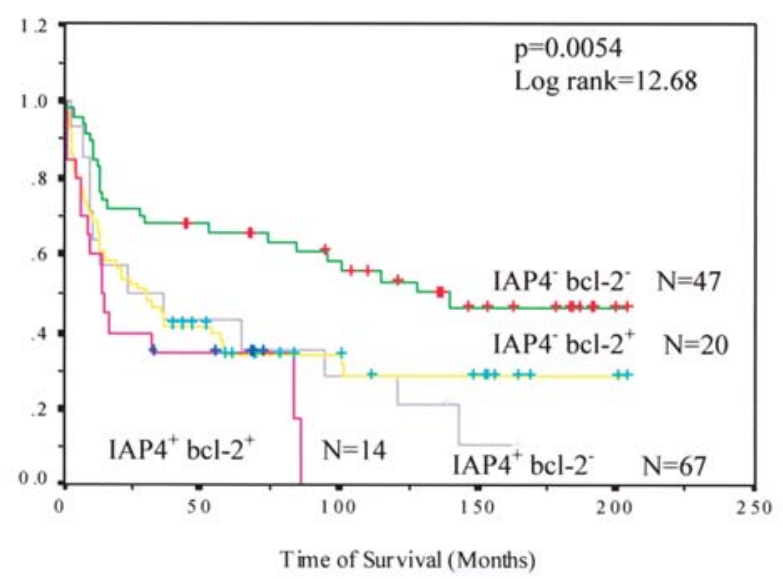

Figure 3. Analysis of patient overall survival based on the expression of IAP-survivin/IAP4 (A and B), bcl-2 (C) or both factors (D). Overexpression of either survivin or bcl-2 predicted a poor disease outcome. (A) Kaplan-Meir survival curve based on the intensity of expression of survivin. (B) Patient survival pattern based on the staining index (see Materials and methods). (D) Patient overall survival time based on the co-expression of IAP-survivin and bcl-2. Cox proportional hazard regression analysis showed that overexpression of both survivin and bcl-2 by the same tumour cells was a more powerful indicator of disease outcome than either factor alone: for survivin $>150$ versus $<150$, relative risk (RR) $=6.20$ (B); for bcl-2 low versus high, $\mathrm{RR}=6.73$ (C); and for overexpression of both survivin and bcl-2 versus low expression of both factors, RR=12.68 (D).

The Mab designated F2-9C3 reacted with the full-length recombinant poly-his-tagged protein, the GST-IAPsurvivin $_{98-142}$ as well as with two proteins present in cell lysates of Jurkat T-cell lymphoma cells and the A549 small cell lung carcinoma cell line. The proteins detected in cell lysates were of molecular weights of approximately 16 and $18 \mathrm{kDa}$, similar to those reported for the full-length native survivin (10) and the survivin $2 \mathrm{~B}$ isoform (16), respectively. These findings indicate that the F2-9C3 antibody identified the native full-length protein as well as the survivin $2 \mathrm{~B}$ isoform and, in addition, that these proteins were expressed in A549 and Jurkat cells confirmed previous reports (10).

The degree of expression varied and both native IAPsurvivin and IAP-survivin 2B were detected in nocodazoletreated A549 cells (G2/M phase arrest; 13) while the proteins were undetectable in untreated cell lysates (data not shown).
By contrast both proteins were detected in untreated Jurkat T-cell lymphoma cells suggesting that survivin is constitutively expressed in these cells, as reported previously (10). As mentioned previously, nocodazole is a drug that introduces a G2/M phase block during cell division (13). Survivin expression increases during mitosis and acts both to complex with and to stabilise the formation of the mitotic spindle (31), a process that requires the inhibition of apoptosis which is aided by survivin (13). The strong staining of the mitotic spindle that we observed reinforces our suggestion that the F2-9C3 Mab identified the native full-length anti-apoptotic protein. Interestingly, an increase in expression of isoform 2B was also evident in nocodazole-treated cells implying that the sole function of this variant may not be as a simple proapoptotic antagonist of the full-length product as has been proposed (16). 
Immunohistochemical staining of normal tissue sections with the F2-9C3 Mab revealed no detectable expression of survivin with the exception of ovary, cerebral cortex and testis which displayed low levels of expression of survivin, corroborating previous findings (32). In normal replicating cells, strong expression of survivin was observed and was specifically associated with the mitotic spindles in accordance with published reports (31). Comparing these findings with staining of neoplastic cells of a variety of tumours examined, also confirms previous reports of the differential distribution of survivin between normal and malignant cells and of survivin being undetectable in non-replicating normal cells $(10,19)$.

Survivin is overexpressed in cancer (19) and has been implicated in tumourigenesis (33). The increased expression of survivin in malignancy may be partly due to the activity of the protein that relates to the $\mathrm{G} 2 / \mathrm{M}$ check-point during mitotic cell division. Survivin may act as an effector molecule at the interface between cell cycle progression and control of apoptosis (13). As mentioned above, during the G2/M phase of mitosis, survivin interacts with the microtubules of the mitotic spindles in a specific and tightly-regulated reaction (34). Disruption of this interaction induces the activation of effector caspases which, in turn, precipitate apoptosis (13). It has been proposed that survivin may counteract a default induction of apoptosis at this check-point in order to preserve cell integrity during cell division (13). De-regulated expression of survivin in individual cells may overcome this check-point and allow progression of aberrant cells through mitosis, a critical step in the oncogenic process.

Sub-cellular localisation and the intensity of the expression of survivin within the various cancer tissues examined in this study varied and staining was observed in the cytoplasm and/ or nuclei as described in previous studies (15, reviewed in ref. 35). With respect to tumour tissue sections from patients with DLBCL, we observed mainly nuclear expression although, in a small number of cases, there was also a weak cytoplasmic staining implying that, at least in DLBCL, expression of survivin rests mainly with the anti-apoptotic type 1 isoform of the molecule which is localised in the nucleus of replicating cells $(13,34)$. By contrast, studies using different antibodies have reported intracytoplasmic expression in patients with DLBCL (21) or other high-grade NHL cases (10). This may reflect the isoform reactivity of different antibodies used in independent IHC studies.

The significance of the sub-cellular localisation of survivin in cancer cells remains unclear. It has been suggested that nuclear expression may be associated with regulation of cell proliferation pathways while cytoplasmic protein may be involved in apoptosis-related events (35). Survivin has been detected in the cytoplasm of tumour cells and therefore it is often regarded as a cytoplasmic protein (10). However, a previous study has reported that survivin is a shuttling protein that is actively exported from the nucleus by a nucleocytoplasmic transport system (36). It can be hypothesised that the active transport of survivin and its variant isoforms between the nucleus and cytoplasm may be one of the mechanisms that regulate the function of these proteins

To date, a great deal of contradictory information from a variety of reports exists regarding the function and the significance of nuclear and cytoplasmic survivin (reviewed in ref. 35). The BIC-5 survivin gene encodes several different protein isoforms, some with opposing apoptotic activities (16) and displaying distinct sub-cellular tropisms (15). Lack of isoform-specific antibodies and the current subjectivity of the methods employed to assess expression may partly explain these conflicting findings. Using the F2-9C3 Mab, we report over-expression of nuclear survivin in patients with DLBCL, suggesting that, at least in DLBCL, our Mab identifies the anti-apoptotic native full-length nuclear protein. We also showed that elevated levels of nuclear protein predict for poor disease outcome. Patients with high nuclear expression experienced a significantly shorter overall mean survival time when compared with those who had low expression. These findings confirm a previous study which also demonstrated that patients with high expression of survivin (70-90\% of cells stained by IHC) displayed a significantly lower 5-year overall survival rate than patients with low expression $(<5 \%)(21)$. However, by contrast to the findings of the present study, Adida et al reported cytoplasmic survivin expression to be of prognostic significance (21). These conflicting findings may be due to the differences in the specificities of the antibodies used. While a Mab was used in the present study, Adida et al used a polyclonal antibody which could have identified a number of different survivin isoforms displaying different localisation.

Further evidence to support the notion that nuclear rather than cytoplasmic survivin may relate to the degree of tumour aggression in high-grade lymphoma is provided by the study of Kuttler et al (37), who used gene expression profiling to show that, in patients with DLBCL, high expression of survivin was associated with a significant increase in the expression of a number of cell cycle activators and that there was a preferential relationship between survivin and cyclin B gene up-regulation and expression. The same authors also reported that low cyclin B mRNA expressors were a characteristic of the germinal centre phenotype which has been reported to identify the group of patients with a good prognosis (37). Taken together, these findings imply that, in DLBCL, overexpression of survivin is associated more avidly with tumour aggression, probably as a result of survivin-mediated cell cycle promotion rather than involvement in anti-apoptotic processes that take place mainly in the cytoplasm (38).

In addition, in Mantle cell lymphoma, a B-cell lymphoma, IHC, Western blotting and real-time PCR revealed nuclear expression of survivin in tumours and also that the expression levels were higher in the blastoid variants and significantly associated with the proliferative capacity of these tumours (39). These findings also highlight the $\mathrm{G} 2 / \mathrm{M}$ transition promotion in this malignancy.

The role of nuclear versus cytoplasmic survivin in highgrade lymphoma requires further investigation as, to date, the function of the various survivin variants with differing apoptotic activities and localisation remains unclear.

With respect to other solid cancers, a large number of studies have also demonstrated that increased levels of nuclear rather than cytoplasmic survivin identify groups of patients with poor prognosis (35). This again implies that the proliferative activity of the malignant cells rather than their prolonged life span may be responsible for tumour 
aggression. By contrast, few studies have reported nuclear expression to be a favourable prognostic marker (35).

Bcl-2 is an anti-apoptotic protein located in the inner mitochondria membrane and it is expressed in a variety of tumours including a proportion of cases of DLBCL (5). Since bcl-2 protein promotes survival and confers a growth advantage on malignant B cells, a number of studies, including the present, have examined the association between bcl-2 expression and disease outcome in patients with DLBCL. We report that elevated levels of bcl-2 protein identify the patients who are at a high risk of disease progression. These findings confirm previous reports $(7-9,40$, reviewed in ref. 41). Interestingly, the present investigation also demonstrated that co-expression of IAP-survivin and bcl-2 had a significantly higher prognostic power than either factor alone. This additive effect highlights the fact that these two factors are localised in different cell compartments and function at different levels in the apoptotic pathways. Thus, while bcl-2 initiates mitochondria-related antiapoptotic events (5), it has been proposed that mitochondriaderived cytosolic survivin is involved in the inhibition of apoptosis in response to cell-death stimuli (38) while nuclear protein controls cell division and proliferation, a process that, as mentioned before, requires the inhibition of apoptosis (13).

In addition, since both bcl-2 and survivin are implicated in the activation of anti-apoptotic pathways and tumourigenesis, both molecules have been evaluated for suitability in anti-cancer therapy. Indeed, overexpression of bcl-2 protein has been incriminated in the resistance of NHL cells to chemotherapy (7,8, reviewed in ref. 41) and bcl-2-specific anti-sense oligonucleotides have shown anti-cancer activities in pre-clinical models (42). With respect to survivin, a number of studies have provided evidence to show that targeting survivin induces apoptosis and re-sensitises cancer cells to chemotherapy (18). Down-regulation of survivin by antisense oligonucleotides co-operates with anti-cancer regimens to facilitate tumour eradication (43) and it may be an attractive therapeutic strategy for patients with aggressive NHL (44).

Exploitation of the survivin and bcl-2 pathways in highgrade lymphoma may provide important information for the determination of prognosis and diagnosis and also offer new therapeutic alternatives, especially for patients who are resistant to current chemotherapy.

\section{Acknowledgements}

The authors would like to acknowledge the support of Marrow \& Stem Cell Trust 2000 and of the Sunderland Blood Disorders Fund for sponsorship of this study.

\section{References}

1. Jin Z and El-Deiry WS: Overview of cell death signalling pathways. Cancer Biol Ther 4: 139-163, 2005.

2. Rudin CM and Thompson CB: Apoptosis and disease: Regulation and clinical relevance of programmed cell death. Annu Rev Med 48: 267-281, 1997.

3. Reed JC: Bcl-2 and the regulation of programmed cell death. J Cell Biol 124: 1-6, 1994

4. Tsujimoto Y, Cossman J, Jaffe E and Croce CM: Involvement of the bcl-2 gene in human follicular lymphoma. Science 228: 1440-1443, 1985.

5. Jaattela M: Escaping cell death: Survival proteins in cancer. Exp Cell Res 248: 30-43, 1999.
6. Sup SJ, Alemany CA, Pohlman B, et al: Expression of bcl-2 in classical Hodgkin's lymphoma: An independent predictor of poor outcome. J Clin Oncol 23: 3773-3779, 2005.

7. Gascoyne RD, Adomat SA, Krajewski S, et al: Prognostic significance of bcl-2 protein expression and bcl-2 gene rearrangement in diffuse aggressive non-Hodgkin's lymphoma. Blood 90: 244-251, 1997.

8. Barrans SL, Carter I, Owen RG, et al: Germinal centre phenotype and bcl-2 expression combined with the International Prognostic Index improves patient risk stratification in diffuse large B-cell lymphoma. Blood 99: 1136-1143, 2002.

9. Colomo L, Lopez-Guillermo A, Perales M, et al: Clinical impact of the differentiation profile assessed by immunophenotyping in patients with diffuse large B-cell lymphoma. Blood 101: 78-84, 2003.

10. Ambrosini G, Adida C and Altieri DC: A novel anti-apoptosis gene, survivin, expressed in cancer and lymphoma. Nat Med 3: 917-921, 1997.

11. Deveraux QL and Reed JC: IAP family proteins - suppressors of apoptosis. Gene Dev 13: 239-252, 1999.

12. Altieri DC: Survivin in apoptosis control and cell cycle regulation in cancer. Prog Cell Cycle Res 5: 447-452, 2003.

13. Li F, Ambrosini G, Chu EY, Plescia J, Tognin S, Marchisio PC and Altieri DC: Control of apoptosis and mitotic spindle checkpoint by survivin. Nature 396: 580-584, 1998.

14. Tamm I, Wang Y, Sausville E, Scudiero DA, Vigna N, Oltersdorf T and Reed JC: IAP-family protein survivin inhibits caspase activity and apoptosis induced by fas (CD95), bax, caspases, and anti-cancer drugs. Cancer Res 58: 5315-5320, 1998.

15. Mahotka C, Liebmann J, Wenzel M, Suschek CV, Schmitt M, Gabbert HE and Gerharz CD: Differential subcellular localization of functionally divergent survivin splice variants. Cell Death Diff 9: 1334-1342, 2002.

16. Mahotka C, Wenzel M, Springer E, Gabbert HE and Gerharz CD: Survivin- $\triangle \mathrm{Ex} 3$ and survivin-2B: Two novel splice variants of the apoptosis inhibitor survivin with different antiapoptotic properties. Cancer Res 59: 6097-6102, 1999.

17. Badran A, Yoshida A, Ishikawa K, Goi T, Yamaguchi A, Ueda T and Inuzuka M: Identification of a novel splice variant of the human anti-apoptosis gene survivin. Biochem Biophys Res Commun 314: 902-907, 2004.

18. Altieri DC: Validating survivin as a cancer therapeutic target. Nat Rev Cancer 3: 46-54, 2003.

19. Fukuda $\mathrm{S}$ and Pelus LM: Survivin, a cancer target with an emerging role in normal adult tissues. Mol Cancer Ther 5: 1087-1098, 2006.

20. Sah NK, Khan Z, Khan GJ and Bisen PS: Structural, functional and therapeutic biology of survivin. Cancer Lett 244: 164-171, 2006.

21. Adida C, Haioun C, Gaulard P, et al: Prognostic significance of survivin expression in diffuse large B-cell lymphoma. Blood 96: 1921-1925, 2000.

22. Sehn LH, Berry B, Chhanabhai M, et al: The revised International Prognostic Index (R-IPI) is a better predictor of outcome than the standard IPI for patients with diffuse large B-cell lymphoma treated with R-CHOP. Blood 109: 1857-1861, 2007.

23. Project TIN-HsLPF: A predictive model for aggressive nonHodgkin's lymphoma. The International Non-Hodgkin's Lymphoma Prognostic Factors Project. N Engl J Med 329: 987-994, 1993.

24. Alizadeh AA, Eisen MB, Davis RE, et al: Distinct types of diffuse large B-cell lymphoma identified by gene expression profiling. Nature 403: 503-511, 2000.

25. Rosenwald A, Wright G, Chan WC, et al: The use of molecular profiling to predict survival after chemotherapy for diffuse large-B-cell lymphoma. N Engl J Med 346: 1937-1947, 2002.

26. Muris JJF, Meijer CJLM, Vos W, vanKrieken JHJM, Jiwa NM, Ossenkoppele GJ and Oudejans JJ: Immunohistochemical profiling based on bcl-2, CD10 and MUM1 expression improves risk stratification in patients with primary nodal diffuse large B cell lymphoma. J Pathol 208: 714-723, 2006.

27. Cameron DA, White JM, Proctor SJ, et al: CHOP-based chemotherapy is as effective as alternating PEEC/CHOP chemotherapy in a randomised trial in high-grade non-Hodgkin's lymphoma. Eur J Cancer 33: 1195-1201, 1997.

28. Lodge AJ, Anderson JJ, Ng SW, Fenwick F, Steward M, Haugk B, Horne CHW and Angus B: Expression of topoisomerase IIIa in normal and neoplastic tissues determined by immunohistochemistry using a novel monoclonal antibody. Br J Cancer 83: 498-505, 2000. 
29. Detre S, Saclani Jotti G and Dowsett M: A 'quickscore' method for immunohistochemical semiquantitation: validation for oestrogen receptor in breast carcinomas. J Clin Pathol 48: 876-878, 1995.

30. Jaffe ES, Harris NL, Diebold J and Muller-Hermelink HK: World Health Organization classification of lymphomas: a work in progress. Ann Oncol 9: S25-S30, 1998.

31. Lens SMA, Vader G and Medema RH: The case for survivin as mitotic regulator. Curr Opin Cell Biol 18: 616-622, 2006.

32. Spaulding B, Pan D, Ghadersohi A, et al: Characterization of the $12 \mathrm{C} 4$ survivin monoclonal antibody and insight into the expression of survivin in human adult tissues. Histopathology 49: 622-633, 2006.

33. Li F: Role of survivin and its splice variants in tumorigenesis. Br J Cancer 92: 212-216, 2005.

34. Hirokawa N: Microtubule organisation and dynamics dependent on microtubule-associated proteins. Curr Opin Cell Biol 6: 74-71, 1994.

35. Li F, Yang J, Ramnath N, Javle MM and Tan D: Nuclear or cytoplasmic expression of survivin: What is the significance? Int J Cancer 114: 509-512, 2005.

36. Rodriquez JA, Span SW, Ferreira CGM, Kruyt FAE and Giaccone G: CRM-1 mediated nuclear export determines the cytoplasmic localization of the antiapoptotic protein survivin. Exp Cell Res 275: 44-53, 2002.
37. Kuttler F, Valnet-Rabier M-B, Angonin R, et al: Relationship between expression of genes involved in cell cycle control and apoptosis in diffuse large B cell lymphoma: a preferential survivin-cyclin B link. Leukemia 16: 726-735, 2002.

38. Altieri DC: The case for survivin as a regulator of microtubule dynamics and cell-death decisions. Curr Opin Cell Biol 18: 609-615, 2006.

39. Martinez A, Bellosillo B, Bosch F, et al: Nuclear survivin expression in Mantle cell lymphoma is associated with cell proliferation and survival. Am J Pathol 164: 501-510, 2004.

40. Hermine $\mathrm{O}$, Haioun $\mathrm{C}$, Lepage $\mathrm{E}$, et al: Prognostic significance of bcl-2 protein expression in aggressive non-Hodgkin's lymphoma. Blood 87: 265-272, 1996.

41. Lossos IS and Morgensztern D: Prognostic biomarkers in diffuse large B-cell lymphoma. J Clin Oncol 24: 995-1007, 2006.

42. Manion MK and Hockenberry DM: Targeting BCL-2-related proteins in cancer therapy. Cancer Biol Ther 2: S105-S114, 2003.

43. Olie RA, Simoes-Wust P, Baumann B, Leech SH, Fabbro D, Stahel RA and Zangemeister-Wittke U: A novel antisense oligonucleotide targeting survivin expression induces apoptosis and sensitizes lung cancer cells to chemotherapy. Cancer Res 60: 2805-2809, 2000.

44. Ansell SM, Arendt BK, Grote DM, et al: Inhibition of survivin expression suppresses the growth of aggressive non-Hodgkin's lymphoma. Leukemia 18: 616-623, 2004. 